\title{
CARACTERIZAÇÃO FÍSICA, QUÍMICA E MINERALÓGICA DO JASPILITO DE CARAJÁS
}

\author{
William Thiago de Sousa da Silva ${ }^{\text {* }}$ \\ Geanso Miranda de Moura ${ }^{2}$ \\ Denilson da Silva Costa' \\ Douglas Martins Sousa ' \\ Roberto Tetsuo Fujiyama ${ }^{3}$
}

\begin{abstract}
Resumo
O jaspilito é uma rocha característica da formação Carajás, composta normalmente por bandamentos de jaspe e minerais de ferro, e que atualmente é classificado como estéril nas minas do complexo Carajás. O objetivo do presente trabalho foi contribuir para o desenvolvimento de possíveis rotas de processamento a fim de possibilitar o aproveitamento econômico do jaspilito de Carajás. Para atingir esse objetivo, foi feita a caracterização física, química e mineralógica do jaspilito: Work Index de Bond para a determinação do consumo energético na moagem, difração de raios- $X$ para determinação e quantificação das espécies minerais presentes, microscopia ótica para observação dos minerais sob luz refletida e análise química por fluorescência de raios-X. O jaspilito analisado apresentou um Work Index superior a $15 \mathrm{kWh} / \mathrm{t}$, teor de 34, $19 \%$ de ferro e as fases minerais identificadas foram quartzo (47,3\%), magnetita $(41,5 \%)$, goethita $(9,0 \%)$ e hematita $(2,2 \%)$. Pelos resultados obtidos, conclui-se que é possível traçar rotas de processamento para o aproveitamento econômico do jaspilito de Carajás.
\end{abstract}

Palavras-chave: Caracterização; Jaspilito; Carajás.

\section{PHYSICAL, CHEMICAL AND MINERALOGICAL CHARACTERIZATION OF JASPILITE FROM CARAJÁS}

\begin{abstract}
The jaspilite is a characteristic rock of the Carajás formation, usually composed of jasper bands and iron minerals, and is currently classified as barren in the mines of the Carajás complex. The objective of this work was to contribute to the development of possible processing routes in order to allow the economic use of Carajás jaspilito. In order to reach this objective, the physical, chemical and mineralogical characterization of the jaspilite was carried out: Bond Work Index for the determination of energy consumption in the milling, X-ray diffraction for determination and quantification of the mineral species present, optical microscopy for minerals observation under reflected light and chemical analysis by $\mathrm{X}$-ray fluorescence. The analyzed jaspilite presented a Work Index superior to $15 \mathrm{kWh} \mathrm{/t,} \mathrm{34,} 19 \%$ iron content and the identified mineral phases were quartz (47.3\%), magnetite (4I.5\%), goethite ( $9.0 \%)$ and hematite $(2.2 \%)$. From the results obtained, it is concluded that it is possible to trace processing routes for the economic use of Carajás jaspilito.
\end{abstract}

Keywords: Characterization; Jaspilite ores; Carajás.

\section{INTRODUÇÃO}

A caracterização tecnológica de minérios é uma etapa fundamental para o correto dimensionamento da rota de processamento de minérios, considerando que são fornecidos os subsídios mineralógicos, texturais e demais informações pertinentes para o processamento mineral [I].
O jaspilito, por conta do teor de ferro muito inferior quando comparado ao minério friável e ao minério compacto, e pela maior dureza, ainda é classificado como estéril nas minas do complexo Carajás, apesar da existência de uma gigantesca quantidade deste material ao longo de toda a

'Universidade Federal do Sul e Sudeste do Pará - UNIFESSPA, Marabá, PA, Brasil. E-mail: willthiago.silva@gmail.com

${ }^{2}$ Instituto Federal do Pará - IFPA, Marabá, PA, Brasil.

${ }^{3}$ Universidade Federal do Pará - UFPA, Belém, PA, Brasil.

2176-1523 (C) 2019 Associação Brasileira de Metalurgia, Materiais e Mineração. Publicado pela ABM. Este é um artigo de acesso aberto distribuído sob os termos da licença Creative Commons CC BY-NC-ND (Attribution-NonCommercial-NoDerivs) - https:// creativecommons.org/licenses/by-nc-nd/4.0/. 
formação Carajás. No entanto, devido a quantidade de ferro presente - segundo Carvalho et al. [2] na faixa de 20 a 40\% - é importante que se ampliem os conhecimentos sobre as propriedades físicas, químicas e mineralógicas desta rocha para um possível aproveitamento futuro.

Considerando a finitude dos recursos minerais e a busca constante pela sustentabilidade, esta investigação tem o intuito de contribuir para o desenvolvimento de possíveis rotas de processamento a fim de possibilitar o aproveitamento econômico do jaspilito de Carajás.

\section{MATERIAIS E MÉTODOS}

\section{I Amostras}

As amostras analisadas neste trabalho (Figura I) foram fornecidas pela empresa Vale S.A., provenientes do Projeto SIID em Canaã dos Carajás, Estado do Pará.

\subsection{Determinação do Work Index (WI) e da Energia Consumida}

Os ensaios para determinação do WI foram realizados em duplicata e de acordo com os procedimentos descritos na NBR I I 376 ABNT [3].

Foi utilizado um moinho de Bond padrão, que possui 12 polegadas de diâmetro, 12 polegadas de comprimento, operando com rotação em torno de 70 RPM, pertencente a mini usina de Tratamento de Minérios da Universidade Federal do Sul e Sudeste do Pará - Unifesspa.

Inicialmente procedeu-se a britagem da rocha até que todo o material fosse passante na malha de 6 mesh (3360 $\mu \mathrm{m})$. Os critérios de parada, cálculo de moabilidade, análise da granulometria da alimentação e do produto foram feitos de acordo com o procedimento padrão. $O \mathrm{~A}_{80}$ e $\mathrm{P}_{80}$ foram obtidos por interpolação.

O valor do $\mathrm{Wl}(\mathrm{kWh} / \mathrm{t})$ foi calculado a partir da Equação I, proposta por Bond [4].

$$
W I=\frac{44,5}{A m^{0,23} \operatorname{Mob}^{0,82}\left(\frac{10}{\sqrt{P}}-\frac{10}{\sqrt{F}}\right)} \times 1,1
$$

onde: Mob = Moabilidade do minério por rotação do moinho de bolas; $\mathrm{Am}$ = Abertura da malha de teste, em micrometros; $\mathrm{P}=$ Abertura, em micrometros, pela qual passa $80 \%$ da

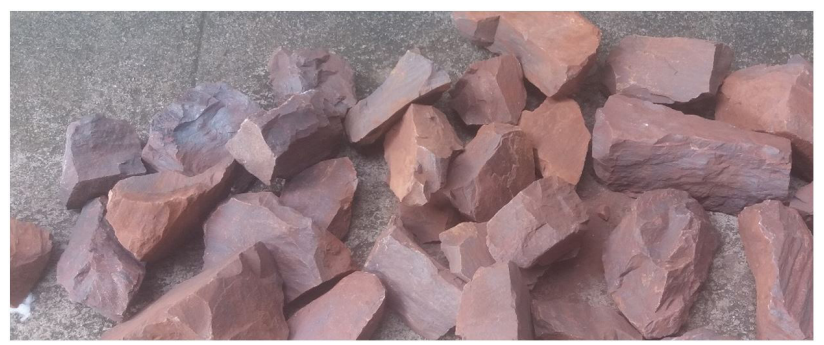

Figura I. Amostras de jaspilito. massa do produto; $\mathrm{F}=$ Abertura, em micrometros, pela qual passa $80 \%$ da massa da alimentação.

O consumo de energia da moagem foi calculado de acordo com a Equação 2, conforme a lei de Bond [4]:

$$
E=10 W I \times\left(\frac{1}{\sqrt{P}}-\frac{1}{\sqrt{F}}\right)
$$

\subsection{Fluorescência de Raios-X}

Para a análise química do jaspilito foi utilizada a técnica da Fluorescência de Raios-X por Energia Dispersiva (FRXED). O equipamento utilizado foi o espectrômetro de Fluorescência de Raios-X da marca Philips, modelo PW-2400, com tubo de anodo de ródio, do Centro de Caracterização de Minerais e Materiais FRX, Santa Luzia - Minas Gerais. Todas as amostras estavam com granulometria abaixo de 200 mesh $(74 \mu \mathrm{m})$. A perda ao fogo foi determinada gravimetricamente por calcinação.

\subsection{Microscopia Ótica}

A partir da amostra, procedeu-se o corte transversal de uma pequena porção para a preparação da seção polida, utilizando a técnica de embutimento. Antes da análise ao microscópio, foi realizado o lixamento grosseiro, o fragmento foi embutido em resina epóxi, foi feito o ataque ácido da superfície e posteriormente o polimento fino. Após a preparação das seções polidas, estas foram submetidas ao microscópio petrográfico ótico de luz transmitida e refletida da marca Zeiss (modelo Axioskop 40), pertencente ao Laboratório de Microscopia Ótica da Universidade Federal do Sul e Sudeste do Pará - Unifesspa. Por se tratar de minerais opacos, foi utilizada a luz refletida em nicóis cruzados.

\subsection{Difração de Raios X}

As amostras foram submetidas a análises por difratometria de raios X, realizadas em Difratômetro de Raios X (DRX) da marca Rigaku, Miniflex 600, que possui tubo de raios $X$ de anodo radiação de $\mathrm{Cu} \mathrm{K} \alpha(\lambda=\mathrm{I} .54 \mathrm{I} 8 \AA$ ) e filtro de Ni para radiações K-beta, pertencente ao Laboratório de Microscopia Ótica da Universidade Federal do Sul e Sudeste do Pará - Unifesspa. A medida foi realizada pelo método do pó, com velocidade de 5 graus por minuto, ângulo de varredura variando de 10 a $110^{\circ}$, passo de $0,02^{\circ}$, goniômetro $\theta-2 \theta$, tensão de trabalho de $40 \mathrm{kV}$ e corrente de $15 \mathrm{~mA}$. A faixa granulométrica utilizada foi $-74+44 \mu \mathrm{m}$.

Através do software PDLX (Rigaku), que compara os valores das distâncias interplanares e das intensidades dos picos nos difratogramas das amostras analisadas com o padrão do banco de dados do ICDD - International Centre for Diffraction Data, foi possível extrair dados referentes as fases minerais presentes no jaspilito.

Além da identificação, foi aplicado o método de Rietveld para quantificação das fases detectadas. 


\section{RESULTADOS E DISCUSSÕES}

\section{I Work Index de Bond e Consumo de Energia na Moagem}

Os resultados dos ensaios de Wl em moinho de bolas estão mostrados na Tabela I.

Aplicando-se a Equação I, foi calculado o Work Index de cada teste:

$$
\mathrm{WI}(\mathrm{I})=15,92 \mathrm{kWh} / \mathrm{t} \text { e WI }(2)=15,58 \mathrm{kWh} / \mathrm{t}
$$

Considerando os valores dos testes realizados com a amostra de jaspilito, o valor final do WI foi determinado através da média aritmética (Equação 3).

$$
W I=\frac{W I(I)+W I(2)}{2}
$$

$\mathrm{WI}=\mathrm{I} 5,75 \mathrm{kWh} / \mathrm{t}$.

Determinado o valor do WI, é possível estimar o consumo energético necessário para moer uma tonelada do jaspilito de Carajás, de um determinado diâmetro de partículas da alimentação até a granulometria $80 \%$ passante em 106 micrometros, através da Equação 2.

$$
E=10,67 \mathrm{kWh}
$$

Portanto, para moer uma rocha de jaspilito à um $\mathrm{P}_{80}$ de 106 micrometros, utilizando moinho de bolas, estima-se um consumo de energia de 10,67 kWh por tonelada.

A Tabela 2, desenvolvida por Napier Munn [5], mostra uma classificação da dureza de minérios de acordo com os valores do Work Index.

O jaspilito, portanto, com base na Tabela 2, pode ser classificado como duro, do ponto de vista de consumo energético na moagem. A moagem é a etapa fundamental de uma usina de beneficiamento, haja visto que é onde há o maior consumo de energia.
Vasconcelos et al. [6] realizou um trabalho com - itabirito compacto do complexo Serra Azul (MG) e obteve Work Index da moagem de $18 \mathrm{kWh} / \mathrm{t}$. A melhor rota de processo para o itabirito compacto foi através de concentração magnética de médio e alto campo, e flotação catiônica reversa.

Gomes [7], em seu trabalho, afirma que para Work Index acima de $15 \mathrm{kWh} / \mathrm{t}$ é benéfico ao empreendimento a prensa de rolos. Na mina do Salobo o WI do cobre é da ordem de $20 \mathrm{kWh} / \mathrm{t}$, sendo que a moagem é operada com rolos de alta pressão HPGR (High-Pressure Grinding Rolls).

Para o jaspilito, apesar de haver grande resistência a cominuição, é possível traçar rotas de processamento para o seu aproveitamento, já que há minérios de maior dureza que são processados atualmente.

\subsection{Análise Química}

A Tabela 3 mostra os resultados da análise química semiquantitativa do jaspilito feita por Fluorescência de raios-X por energia dispersiva.

O resultado da análise química por fluorescência de raios-X ratifica a elevada pureza dos jaspilitos de Carajás mencionada por Macambira [8], pois, do total, aproximadamente $97 \%$ da composição compreende quartzo e minerais de ferro. Macambira [8], em seu trabalho, realizou a análise química em 62 amostras de rocha dos jaspilitos de Carajás por espectrofotometria, obtendo valores médios $53,65 \%$ de $\mathrm{Fe}_{2} \mathrm{O}_{3} \mathrm{~T}$ e $44,60 \% \mathrm{SiO}_{2}$, semelhantes aos do presente trabalho.

$\mathrm{Na}$ análise química em amostras do jaspilitos de Carajás, Abreu [9] contatou que "Fe e Si no JF somam mais de $98 \%$ do total, com teor de $\mathrm{SiO}_{2}$ variando de $42,6 \mathrm{I}$ a $62,5 \mathrm{I} \%$ e o $\mathrm{Fe}_{2} \mathrm{O}_{3} \mathrm{~T}$ de 35,92 a $56,48 \%$."

O teor de ferro da amostra de jaspilito analisada

\begin{tabular}{|c|c|c|c|c|}
\hline Teste & $\begin{array}{c}\text { Moabilidade } \\
\text { (gramas / rotação) }\end{array}$ & $\mathrm{A}_{80}(\mu \mathrm{m})$ & $\left.\mathbf{P}_{80} \mu \mathrm{m}\right)$ & Malha de teste $(\mu \mathrm{m})$ \\
\hline Primeiro teste $-\mathrm{WI}(I)$ & 1,55 & 1971,40 & 122,64 & 150 \\
\hline Segundo teste - WI (2) & 1,57 & |93,5| & 120,54 & 150 \\
\hline
\end{tabular}
foi de $34,19 \%$, e teor de sílica de 47,82\%. Estes números

Tabela I. Parâmetros do Work Index determinados

Tabela 2. Classificação do material com base no WI

\begin{tabular}{ccccc}
\hline Classificação & Leve & Médio & Duro & Muito duro \\
\hline Valor do Wl $(\mathrm{kWh} / \mathrm{t})$ & 7 a 9 & 9 a I4 & 14 a 20 & Acima de 20 \\
\hline
\end{tabular}

Fonte: Napier Munn et al. [5].

Tabela 3. Análise química do jaspilito

\begin{tabular}{cccccccc}
\hline Gravimetria & \multicolumn{7}{c}{ Fluorescência de raios-X } \\
\cline { 2 - 9 } PPC $1000{ }^{\circ} \mathbf{C}$ & $\mathbf{S i O}_{2}$ & $\mathbf{F e}$ & $\mathbf{A l}_{2} \mathbf{O}_{3}$ & $\mathbf{C a O}$ & $\mathbf{C r}_{2} \mathbf{O}_{3}$ & $\mathbf{M n}$ & $\mathrm{TiO}_{2}$ \\
\hline 2,31 & 47,82 & 34,19 & 0,49 & 0,09 & 0,08 & 0,04 & 0,02 \\
\hline
\end{tabular}


convergem com os dados de Carvalho et al. [2], que denomina o jaspilito como um dos tipos encontrados na província mineral de Carajás com bandamento definido por jaspe e minerais opacos, com teor de ferro entre $20 \%$ e $40 \%$ e de quartzo entre $38 \%$ e $60 \%$.

\subsection{Microscopia Ótica por Luz Refletida (MOLR)}

As fotomicrografias obtidas a partir do microscópio ótico podem ser visualizadas nas Figuras 2, 3 e 4 .

A rocha apresenta bandamento composicional que se dá entre bandas de minerais opacos e minerais silicosos visto no microscópio de luz refletida. A rocha apresenta bandamento regular e persistente lateralmente.

A espessura das bandas de minerais silicosos e opacos são semelhantes e há uma laminação interna com laminas de sílica dentro da banda de opacos, ao mesmo tempo que há laminas de opacos dentro da camada de silicosos. O contato entre as bandas é reto, com poucas ondulações e brusco. Ou seja, com limites bem definidos.

Quanto aos minerais presentes nas bandas, vale ressaltar que na luz refletida os minerais opacos refletem mais a luz incidente na superfície e por isso são mais claros, enquanto os silicosos são os mais escuros porque parte da luz atravessa estes minerais que são translúcidos. Desta forma, considerando a reflectância e o brilho característico de cada mineral sob o microscópio, os minerais da banda silicosa são compostos por chert e jaspe, ambos compostos basicamente por quartzo. O jaspe possui impregnações de ferro, o que lhe dá cor avermelhada. O chert não possui impregnações e é composto por microcristais. Quanto a banda de minerais opacos, constituem minerais de ferro sendo a magnetita e/ou maghemita e hematita em sua maioria. Vale ressaltar que magnetita e maghemita são minerais de mesma composição química e de difícil distinção em microscópio ótico.

\subsection{Difração de Raios-X}

A Figura 5 apresenta o difratograma e os dados do refinamento por Rietveld da amostra. As quantidades que estimam a confiabilidade do ajuste dos dados neste método são: $R_{w p}$ e $R_{p}$ que representam valores em torno de $34 \%$ e $25 \%$, respectivamente, e Goodness of fit (GOF) de 2,06. Para isso, foram usadas as seguintes fases cristalográficas: magnetita (COD 9002332), quartzo (COD I0I 1097), goethita (COD 9003077) e hematita (COD 90I5065). Os parâmetros refinados no software PDXL foram: fator de escala, largura de pico a meia altura (FWHM), assimetria e orientação preferencial. A Tabela 4 mostra o quantitativo das fases minerais presentes na amostra, respectivamente, após o refinamento.

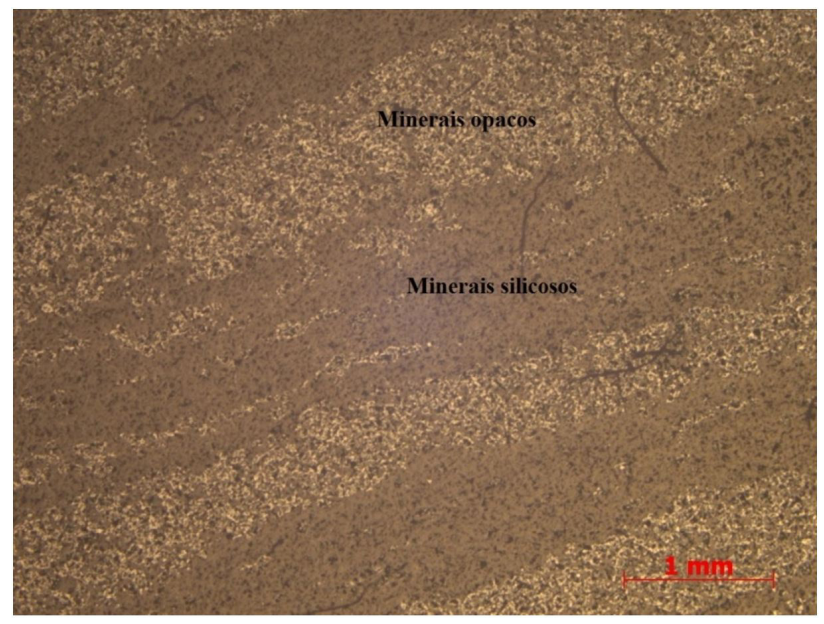

Figura 2. Fotomicrografia ampliada do bandamento composicional regular do jaspilito.

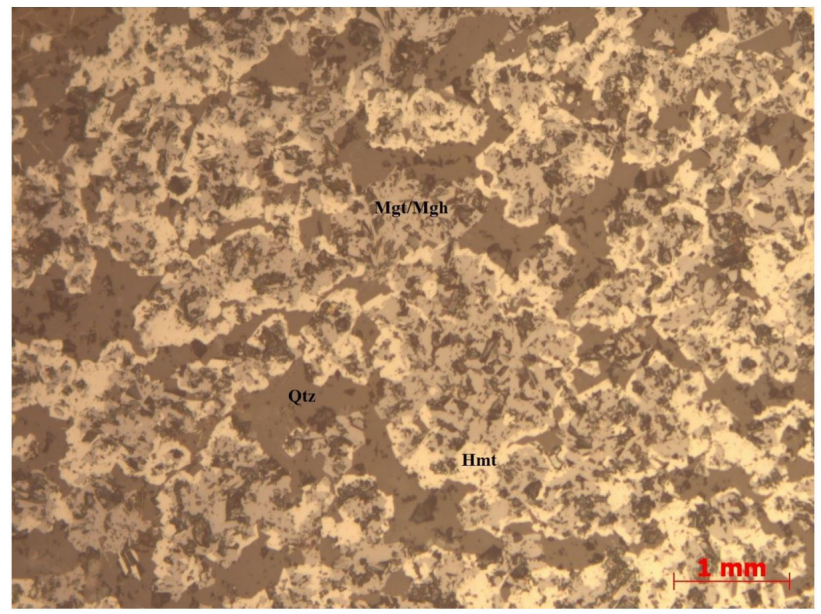

Figura 3. Fotomicrografia ampliada (escala em $0, \mathrm{I} \mathrm{mm}$ ) da banda de minerais opacos (Mgt/Mgh: Magnetita ou Maghemita; Hmt: Hematita; Qtz: Quartzo).

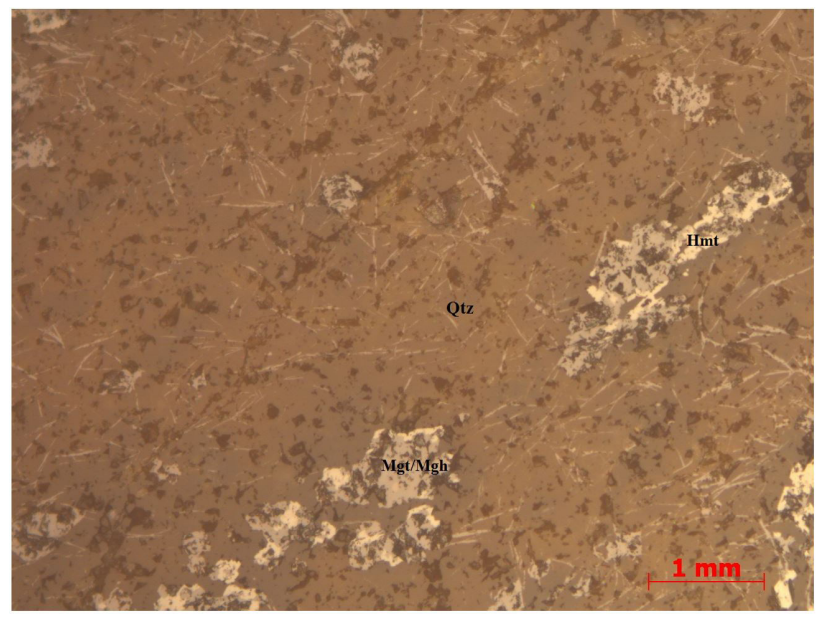

Figura 4. Fotomicrografia ampliada (escala em $0,1 \mathrm{~mm}$ ) da banda de minerais silicosos (Mgt/Mgh: Magnetita ou Maghemita; Hmt: Hematita; Qtz: Quartzo). 


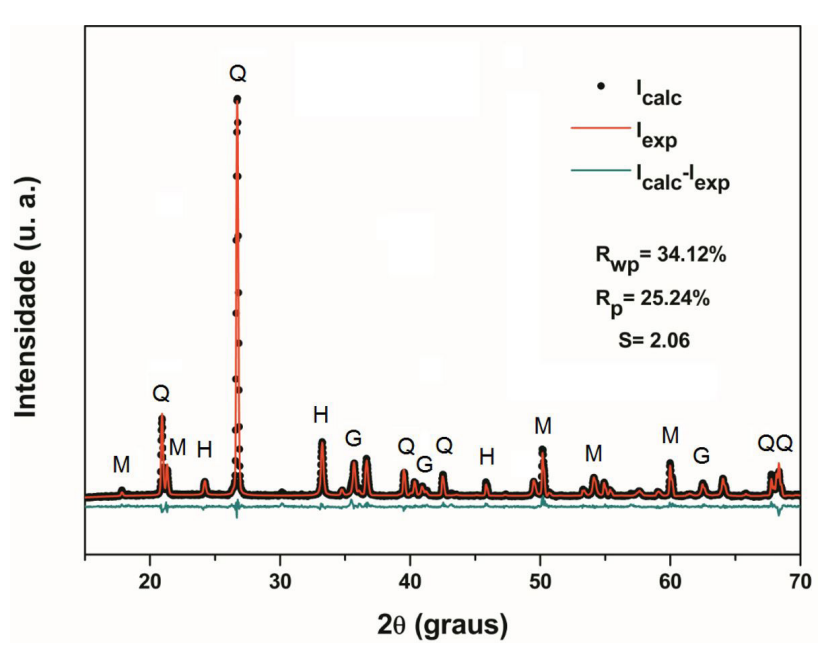

Figura 5. Difratograma do Jaspilito ( $M$ : magnetita, Q: quartzo, $\mathrm{H}$ : hematita, G: goethita). Radiação $\mathrm{Cu} \mathrm{K \alpha}$.

Tabela 4. Quantificação das fases minerais por Rietveld

\begin{tabular}{cccc}
\hline \multicolumn{4}{c}{ Fases Minerais do Jaspilito } \\
\hline Quartzo & Magnetita & Goethita & Hematita \\
\hline 47,3 & 41,5 & 9,0 & 2,2 \\
\hline
\end{tabular}

Observa-se que a amostra apresenta abundância em quartzo e magnetita, juntamente com outras fases em menores quantidades (goethita e hematita). Alguns picos não obtiveram identificação correspondente na leitura do software e por este motivo estão sem marcação no difratograma (Figura 5).

Dentre os minerais ferrosos constatou-se elevado teor de magnetita $(4 I, 5 \%)$ sendo bem superior a goethita e hematita. Quanto ao quartzo, os $47,3 \%$ que compõem a amostra são basicamente chert e jaspe.

Os dados corroboram com a análise química e com a microscopia ótica.

\section{CONCLUSÕES}

OWork index da moagem do jaspilito de Carajás foi de $15,75 \mathrm{kWh} / \mathrm{t}$. Isto representa um valor alto, sendo, portanto, classificado como material duro, com base na tabela desenvolvida por Napier Munn. No entanto, na indústria mineral há minérios mais resistentes a moagem, que demandam maior energia para que sejam fragmentados. Estima-se, por sua vez, que a energia necessária para cominuir uma tonelada de jaspilito abaixo de 106 micrometros é de 10,67 kWh.

A análise química realizada por fluorescência de raios-X indicou teores de quartzo de $47,82 \%$ e $34,19 \%$ do elemento $\mathrm{Fe}$, corroborando com a análise por difração de raios-X. Há outros elementos traços presentes no jaspilito, sendo eles alumínio, cromo, manganês e titânio, ambos oxidados, mas não foram detectadas estas fases nas análises por difração de raios-X. Há outros elementos com teores extremamente baixos $(\mathrm{Na}, \mathrm{Mg}, \mathrm{K}$ e $\mathrm{P})$, inferiores a detecção do aparelho de fluorescência de raios-X utilizado.

Os resultados microscopia óptica de luz refletida do jaspilito de Carajás mostram que a composição do material rochoso é dividida em minerais silicosos e opacos. Os minerais silicosos representam a maior porção dentre os constituintes, sendo basicamente quartzo em duas formas: jaspe (ślica com impregnações de ferro) e chert (sílica em microcristais sem contaminação) em menor quantidade. Os minerais opacos são minerais de ferro diversos, sendo que na difração de raios- $X$ as fases minerais detectadas foram magnetita, goethita e hematita, tendo destaque a elevada porção de magnetita e a baixíssima de hematita, estimados em $41,5 \%$ e 2,2\%, respectivamente.

Pelos teores de ferro e quartzo apresentados, e pela composição mineralógica, apesar de haver certa resistência a cominuição observada no WI de Bond, é possível traçar rotas de processamento para o aproveitamento econômico do jaspilito de Carajás, já que há minérios de maior dureza que são processados atualmente. Britagem em estágios (primária, secundária, terciarias e quaternária), moagem com rolos de alta pressão (HPGR), separação magnética e flotação catiônica reversa são processos a serem analisados nas possíveis rotas de processamento.

\section{REFERÊNCIAS}

I Neumann R, Schneider CL, Alcover A No. Caracterização tecnológica de minérios. In: Luz AB. Tratamento de minérios. 5. ed. Rio de Janeiro: CETEM; 2010. p. 85-137.

2 Carvalho PSL, Silva MM, Rocio MAA, Moszkowicz ]. Minério de ferro. BNDES; 2017 [acesso em 15 nov. 2017]. Disponível em: https://web.bndes.gov.br/bib/jspui/bitstream/1408/4802/I/BS\%2039\%20min\%C3\%A9rio\%20de\%20ferro_P.pdf

3 Associação Brasileira de Normas Técnicas. NBR I I376. Rio de Janeiro: ABNT; 1990.

4 Bond FC. The third theory of comminution. Transaction AIME. 1952; 193:484-494.

5 Napier Munn TJ, Morrell S, Morrinson RD, Kojovic T. Mineral cominuition: their operation and optmisation. Queensland: Julius Kruttschnitt Mineral Research Centre ; 1999. p. 63.

6 Vasconcelos LA, Brandão PRG, Lemos LN. Estudos de caracterização e concentração de itabirito compacto do Complexo Serra Azul, MG. Associação Brasileira de Materiais Metalurgia e Mineração; 2012 [acesso em 10 out. 2017]. Disponível em: http://www.tecnologiammm.com.br/files/v9n2/v9n2a02.pdf. 
Silva et al.

7 Gomes FB. A prensa de rolos como alternativa para os circuitos de cominuição nas atividades de mineração e a viabilidade de sua aplicação [dissertação]. UFMG; 2010.

8 Macambira JB. O ambiente deposicional da Formação Carajás e uma proposta de modelo evolutivo para a Bacia Grão Pará [tese]. Campinas: Instituto de Geociências, Universidade Estadual de Campinas; 2003.

9 Abreu SS. Petrografia e geoquímica de formações ferríferas bandadas e a gênese de cavernas da serra norte, Carajás, Pará [dissertação]. UFPA; 2017.

Recebido em: 23 Mar. 2018

Aceito em: 20 Set. 2018 